\title{
Aprendizaje basado en proyectos colaborativos de entornos de programación a partir de proyectos de ingeniería civil
}

\author{
Collaborative Project-Based Learning of Environments Programming From Civil \\ Engineering Projects
}

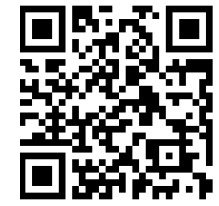

\author{
Rodrigo Fernando Herrera ${ }^{1}$ \\ Pontificia Universidad Católica de Valparaíso \\ Valparaíso, Chile \\ rodrigo.herrera@pucv.cl \\ http://orcid.org/0000-0001-5186-3154
}

Recibido 29 de febrero de 2016 • Corregido 18 de marzo de 2017 • Aceptado 16 de abril de 2017

\begin{abstract}
Resumen: El objetivo de este trabajo es presentar y respaldar la aplicación de una metodología de enseñanza basada en el aprendizaje activo en un curso de programación dictado a estudiantes de primer año de la carrera de ingeniería civil. Las técnicas de aprendizaje activo utilizadas son el aprendizaje basado en proyectos y el aprendizaje colaborativo. La metodología de investigación es el estudio de tres casos. La aplicación de esta metodología se respalda con el alza de los porcentajes de logro de los resultados de aprendizaje obtenidos por el estudiantado de un mismo curso, al cual se le aplicó metodología (curso académico 2015), en desmedro de grupos de estudiantes en donde se aplicó una metodología tradicional de enseñanza (curso académico 2013-2014). Para evaluar el logro se aplicaron evaluaciones formativas y sumativas por la parte docente (heteroevaluación); además, se aplicó una autoevaluación al final del curso. De los resultados obtenidos se evidencia que, cuando se aplica una metodología activa, el estudiantado aumenta su logro en cada resultado de aprendizaje; disminuye la brecha entre su percepción de lo aprendido y la evaluación del profesorado; mejora sus habilidades actitudinales (autoaprendizaje, trabajo en equipo, desarrollo y presentación de informes), entre otras ventajas.
\end{abstract}

Palabras claves: Aprendizaje activo; aprendizaje colaborativo; metodología de enseñanza; evaluación sumativa, autoevaluación.

1 Profesor de la Escuela de Ingeniería Civil de la Pontificia Universidad Católica de Valparaíso, en los cursos de Informática Aplicada, Planificación y Control de Proyectos, y Administración de Proyectos. Desde este año estudiante de Doctorado en Ciencias de la Ingeniería en la Pontificia Universidad Católica de Chile. Dentro de mis líneas de investigación y desarrollo se encuentra la educación en ingeniería y la dirección de proyectos de infraestructura. 
doi: http://dx.doi.org/10.15359/ree.21-2.10

URL: http://www.una.ac.cr/educare

CORREO: educare@una.cr

\begin{abstract}
The objective of this research is to present and support the implementation of a teaching methodology based on active learning in a programming course taught to students of the first year of the civil engineering career. The active learning techniques used are project-based learning and collaborative learning. The research methodology is the study of three cases. The application of this methodology is supported by the increase in the percentages of achievement of the learning results obtained by the students of the same course in which this methodology was implemented (academic year 2015), to the detriment of students where a traditional methodology of teaching was applied (academic year 2013-2014). To evaluate the achievement, formative and summative evaluations were used by the teaching staff (heteroevaluation), and a self-assessment was implemented at the end of the course as well. From the obtained results it is evident that, when applying an active methodology, the students increase their achievement in each learning result; they reduce the gap between their perception of what is learned and the evaluation of teachers; and they improve their attitudinal skills (self-learning, teamwork, development and presentation of reports), among other benefits.
\end{abstract}

Keywords: Active learning; collaborative learning; teaching methodology; summative evaluation; self-assessment

\title{
Introducción
}

Distintos estudios destacan que unas de las habilidades más significativas al momento de encontrar trabajo, permanecer y escalar posiciones en él es la habilidad de trabajar en equipo, liderarlos y poseer una alta capacidad de autoaprendizaje (Martínez, 2011). Esto se puede evidenciar en las encuestas realizadas en el año 2014 a exalumnos y exalumnas de la Carrera de Ingeniería Civil de la Pontificia Universidad Católica de Valparaíso (PUCV) y a sus respectivas personas empleadoras, donde manifiestan que unas de las competencias más destacadas fueron la capacidad de autoaprendizaje y la habilidad de trabajar en equipo (Pontificia Universidad Católica de Valparaíso, PUCV, 2015). Dado este diagnóstico, el personal docente de pregrado es quien debe hacerse responsable de fomentar al estudiantado estas habilidades tan requeridas por el mercado laboral (PUCV, 2015). Unas de las herramientas más potentes para fomentar estas competencias están asociadas al aprendizaje colaborativo y al correcto uso de distintas tecnologías de la información.

El aprendizaje colaborativo se define como un sistema de interacciones cuidadosamente diseñado que organiza la influencia recíproca entre las personas integrantes de un equipo (Johnson y Johnson, 2000). En el caso específico de un curso de pregrado, quienes integran el equipo sería el propio estudiantado, y el profesorado es quien guiará el desarrollo del programa. Este último es el encargado de diseñar un sistema en donde sus estudiantes sean protagonistas de su propio aprendizaje y, a partir de este, sean capaces de influenciarse entre sí, y adquirir mayor comprensión de los contenidos y mayor logro de los resultados de aprendizaje del curso (Serrano y Calvo, 1994). Uno de los factores clave para fomentar la capacidad de autoaprendizaje en el estudiantado es la capacidad de manejar diversas tecnologías de la información útiles para este objetivo.

2

Los artículos de la Revista Electrónica Educare del Centro de Investigación y Docencia en Educación de la Universidad Nacional, Costa Rica, se comparten bajo términos de la Licencia Creative Commons: Reconocimiento, No Comercial, Sin Obra Derivada 3.0 Costa Rica. Las autorizaciones adicionales a las aquí delimitadas se pueden obtener en el correo: educare@una.cr 
Debido a las habilidades de autoaprendizaje y manejo de tecnologías de la información que los grupos profesionales necesitan en el mundo laboral, la educación superior tiene una misión importante en la formación de estas competencias. En las universidades existe una gran diversidad de formas de ejercer la docencia, pero que al final se pueden dividir en dos grupos: la metodología tradicional y la metodología activa participativa.

La metodología tradicional es aquella en la que se da"un enfoque disciplinar, centrado en el aprendizaje conceptual donde el profesor es el centro de desarrollo de la clase (transmisión verbal) y en la que los alumnos solamente actúan como receptores, siendo memorístico el aprendizaje propagado y cuyos únicos recursos empleados son la explicación de profesor, [un libro y la pizarra o presentación de diapositivas]" (Freire, 2014, p. 8).

Por su parte, el aprendizaje activo da pie a un cambio de paradigma en la educación superior, explicita claramente que la planificación de la enseñanza no se orienta solamente hacia los contenidos y metas que se presentan al estudiantado, sino que también hacia estudiantes y sus procesos de adquisición y construcción de conocimientos (Huber, 2008). La metodología de aprendizaje activa es aquella que tiene un enfoque globalizador centrado en el desarrollo de capacidades generales, a través de procedimiento y actitudes científicas. En esta metodología el profesorado es coordinador de la clase y el estudiantado ocupa el papel central (Tyler, 1949). Existen experiencias que respaldan la efectividad del aprendizaje basado en proyecto colaborativos en la educación superior y en el uso de esta práctica como estrategia formativa (Maldonado, 2008; Maldonado y Rojas, 2008).

Este estudio busca ser una evidencia de que las metodologías de aprendizaje activo logran que sus estudiantes logren un aprendizaje más profundo en el uso de las tecnologías de la información, en desmedro de las metodologías de aprendizaje tradicionales, además se busca evidenciar que estas técnicas aportan en el desarrollo de habilidad actitudinales, tales como, el autoaprendizaje y el trabajo en equipo.

En este artículo se presenta cómo se aplicó la metodología de enseñanza activa, específicamente el aprendizaje colaborativo y el aprendizaje basado en problemas (en este caso proyectos de ingeniería). Además, se realiza una comparación entre la metodología tradicional y la metodología activa, con respecto al logro de los resultados de aprendizaje obtenido por estudiantes de un curso particular. Para evidenciar el logro se realizó una heteroevaluación (docente evalúa a estudiantes) y una autoevaluación (estudiantes se evalúan a sí mismos o mismas).

\section{Metodología}

La metodología de investigación que se utilizó es el estudio de casos, particularmente se analizan tres casos: el primero corresponde a un grupo de estudiantes del curso académico 2013; el segundo, a un grupo de estudiantes del curso 2014, y el tercero, a un grupo de estudiantes del curso académico 2015. Los tres casos corresponden a una misma asignatura (informática aplicada), con 
doi: http://dx.doi.org/10.15359/ree.21-2.10

URL: http://www.una.ac.cr/educare

CORREO: educare@una.cr

la diferencia de que en los grupos del curso 2013 y 2014 se aplicó una metodología de enseñanza tradicional, mientras que el curso 2015 se desarrolla con una metodología activa, basada en la realización de proyectos colaborativos. A continuación, se explica cómo se llevó cada uno de los cursos y cómo se logró evidenciar diferenciar en la realización de los dos tipos de metodología.

\section{Contextualización del curso}

Este curso en particular tiene el nombre "CIV 110, Informática Aplicada", y manifiesta su propósito de desarrollar, en sus estudiantes, la capacidad de reconocer, utilizar y aplicar herramientas computacionales para resolver problemas de ingeniería. En esta asignatura, se espera que el estudiantado aplique principios de lógica, conjuntos, física y matemáticas básicas en la resolución de problemas de ingeniería, al diseñar un modelo algorítmico que posteriormente lo implementarán en un programa de computadora. El público objetivo de este curso son estudiantes de segundo semestre de la carrera de Ingeniería Civil de la PUCV, quienes, para poder cursarlo, deben haber aprobado los ramos de Introducción a la Ingeniería y Álgebra (Pontificia Universidad Católica de Valparaíso, Escuela de Ingeniería Civil, 2014).

Los contenidos de este curso se dividen en tres unidades: Fundamentos de algoritmia, programación en MS Excel y programación en MATLAB. Y los resultados o logros de aprendizajes asociados a estas unidades son:

1. Aplicar y utilizar técnicas de formulación y diseños de algoritmos.

2. Comprender y desarrollar estructuras algorítmicas para resolver problemas de ingeniería.

3. Adquirir habilidades de manejo y análisis de datos en MS Excel.

4. Adquirir el dominio básico del manejo y análisis de datos en MATLAB.

5. Generar informes de ingeniería mediante el análisis de datos.

6. Utilizar herramientas como MS Excel y MATLAB para la resolución de problemas de ingeniería.

En promedio, esta asignatura tiene cerca de cincuenta estudiantes, un profesor o profesora de cátedra y dos ayudantes (estudiantes de curso superior) que apoyan con la realización de talleres. Históricamente las clases de cátedra son un salón tradicional con puestos de trabajo para cada estudiante (espacio solo para cuaderno), una pizarra acrílica y proyector de computadora. Una consideración importante es el número de estudiantes que se tuvo en cada curso: 55 estudiantes el 2013; 41 el 2014, y 61 el 2015. Si bien este número no varía considerablemente año tras año, el número que recibe cada año hace que se pueda considerar como un curso masivo y, por lo tanto, la dificultad de aplicar metodologías de aprendizaje activo se convierte en un gran desafío (Biggs \& Tang, 2011). 


\section{Evaluación de los resultados de aprendizaje}

Para efectos de este estudio se consideran dos grupos de estudio: el primero integrado por estudiantes del curso en 2013 o 2014, a quienes se les aplicó una metodología de aprendizaje tradicional y el segundo grupo son estudiantes del 2015 a quienes se les aplicaron las metodologías de aprendizaje basado en proyectos y colaborativo.

Para medir los resultados de la heteroevaluación y autoevaluación se tomaron las muestras que se presentan en la Tabla 1 para cada agente evaluativo.

Tabla 1: Muestra de estudiantes para hetero y autoevaluación

\begin{tabular}{ccc}
\hline Generaciones & $\begin{array}{c}\text { Muestra heteroevaluación } \\
\text { (Muestra = Población) }\end{array}$ & Muestra autoevaluación \\
\hline $2013-2014$ (Metodología tradicional) & 96 & 48 \\
2015 (Aprendizaje activo) & 61 & 53 \\
\hline
\end{tabular}

Se debe considerar que para la heteroevaluación de los años 2013 y 2014 se ocuparon pruebas, controles y sus respectivas pautas; mientras que para el 2015 se utilizaron controles, tareas, proyectos con sus respectivas rúbricas y escalas de apreciación, según fuera el caso. Para ambos grupos se considera una escala de logro entre 0 a $100 \%$.

Para hacer la autoevaluación, los grupos de estudiantes contestaron un instrumento que tenía dos secciones: Escala de apreciación (1 a 5) de logro de cada uno de los resultados de aprendizaje del curso; y una segunda parte de preguntas abiertas en donde se les pedía una opinión acerca de la asignatura.

\section{Metodología de aprendizaje activo 2015}

Para desarrollar esta metodología se dividió el proceso en cuatro etapas: la primera es de planificación y preparación del material y recursos de apoyo del curso; la segunda corresponde a la de ejecución misma de la metodología; la tercera etapa consiste en la de evaluación y seguimiento del proceso de aprendizaje; y la última fase es la de conclusiones y obtención de lecciones aprendidas para la próxima realización del curso. En la Figura 1 se explica la secuencia traslapada de cada una de las etapas de la aplicación de la metodología. 
doi: http://dx.doi.org/10.15359/ree.21-2.10

URL: http://www.una.ac.cr/educare

CORREO: educare@una.cr

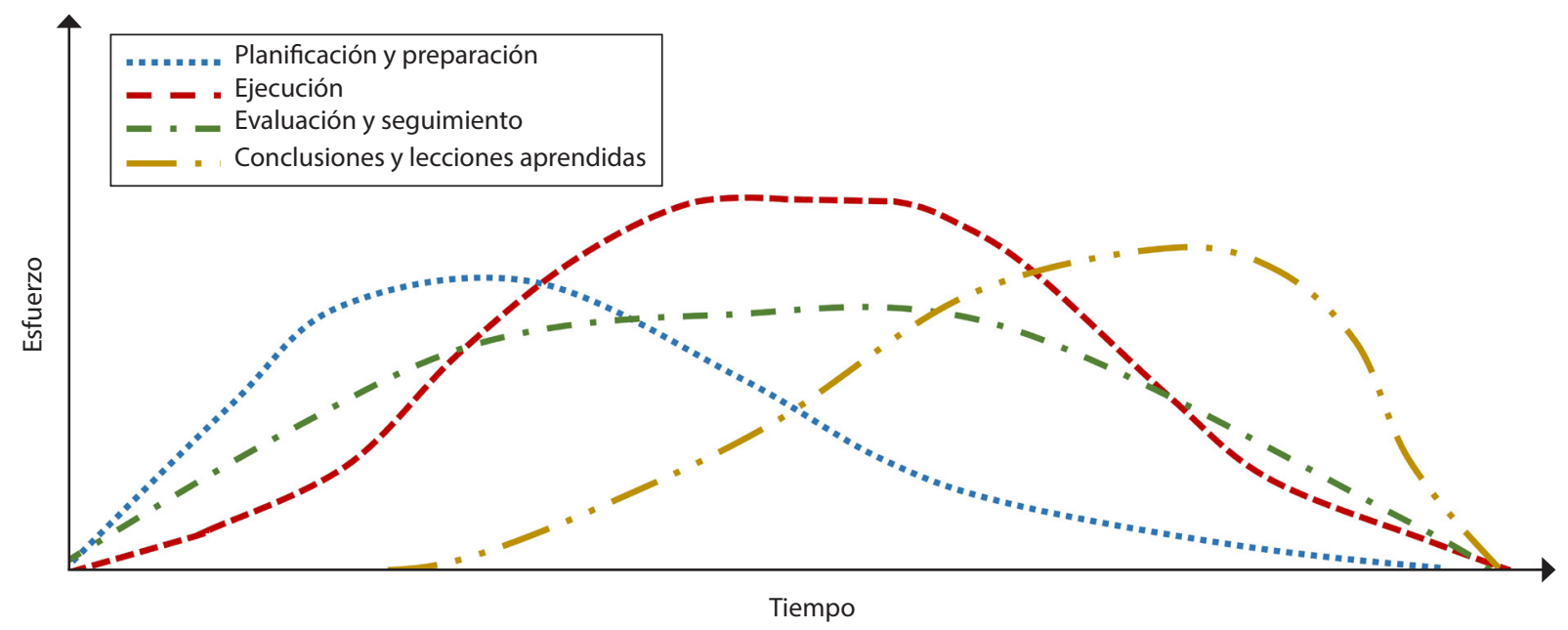

Figura 1: Nivel de esfuerzo en el tiempo de cada etapa de la metodología de aplicación.

\section{Planificación y preparación del material y recursos de apoyo}

La planificación del curso consiste de tres etapas fundamentales: la planificación global, el diseño de cada clase en particular y la preparación de los recursos de apoyo del aprendizaje.

La planificación global se realizó considerando las unidades de aprendizaje, los resultados de aprendizaje asociados, la duración del curso ( 28 sesiones de cátedra de 2 horas pedagógicas cada una y 26 sesiones de taller de 2 horas pedagógicas cada una); además, dentro de las clases de cátedra se agregó un indicador que busca diferenciar entre clases prácticas/teóricas y clases netamente prácticas (Ver Figura 2).

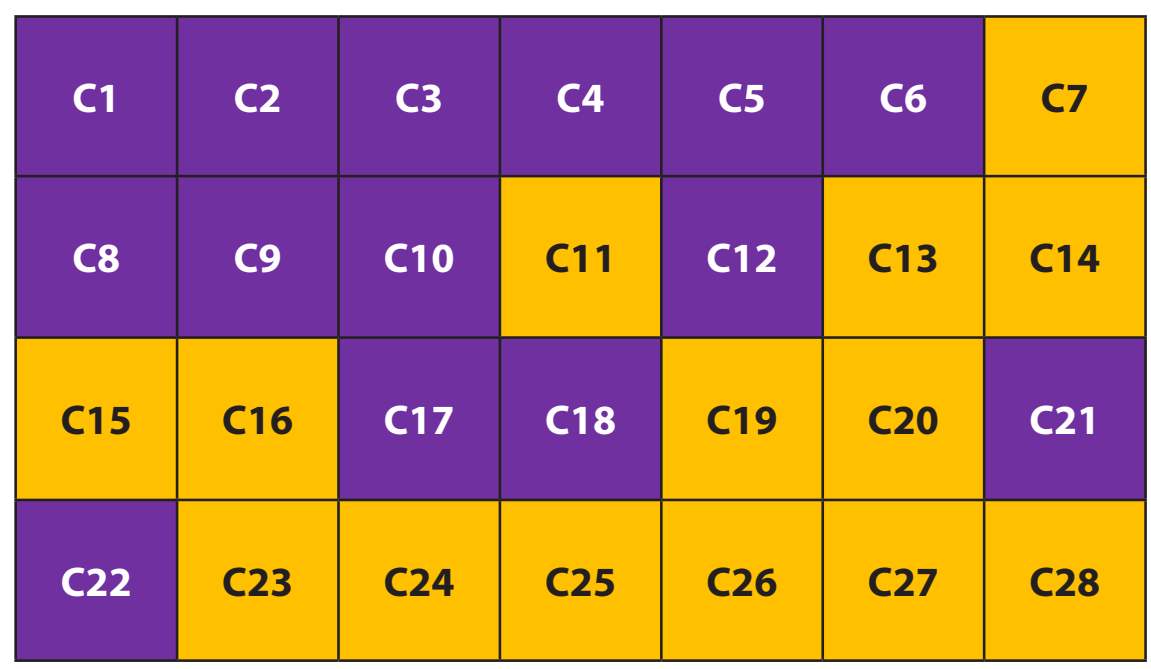

Clase práctica/teórica

Clase práctica

Figura 2: Planificación global de clases prácticas y clases prácticas/teóricas del curso. 
El diseño de cada clase en particular sigue una estructura de inicio, desarrollo y cierre. En las clases todas las sesiones el desarrollo se descomponen en las siguientes subetapas: introducción a los contenidos nuevos (ICN), práctica guiada (PG), y práctica independiente (PI). Cada una de estas etapas y subetapas se describe con mayor precisión en la Tabla 2. En las sesiones del tipo práctica/teórica la repartición temporal de las tres subetapas ICN, PG y PI es bastante homogénea, a diferencia de las clases netamente prácticas, en donde la ICN se reduce considerablemente, tomando todo el protagonismo de la sesión la PG y la PI.

Tabla 2: Etapas y subetapas de la clase

\begin{tabular}{|c|c|}
\hline Etapa y/o subetapa & Preguntas que debe responder el personal docente para planificar cada etapa de la clase \\
\hline \multirow{5}{*}{ Inicio } & • ¿Cómo comunicará lo que está a punto de suceder? \\
\hline & • ¿Cómo comunicará el cómo sucederá? \\
\hline & • ¿Cómo comunicará su importancia? \\
\hline & • ¿Cómo comunicará las conexiones con las clases previas? \\
\hline & • ¿Cómo comprometerá a sus estudiantes y capturará su interés? \\
\hline \multirow{5}{*}{$\begin{array}{l}\text { Desarrollo - Introducción } \\
\text { a los contenidos nuevos }\end{array}$} & $\begin{array}{l}\text { - ¿Cómo explicará/demostrará todos los conocimientos/habilidades necesarias del } \\
\text { objetivo, para que sus estudiantes comiencen a internar activamente los puntos claves? }\end{array}$ \\
\hline & • ¿Qué potenciales malentendidos anticipa? \\
\hline & • ¿Cómo los mitigará proactivamente? \\
\hline & • ¿Cómo/cuándo revisará la comprensión? \\
\hline & ¿Cómo enfrentará los malentendidos? \\
\hline \multirow{4}{*}{$\begin{array}{l}\text { Desarrollo - Práctica } \\
\text { guiada }\end{array}$} & $\begin{array}{l}\text { - ¿Cómo practicarán sus estudiantes todos los conocimientos/habilidades } \\
\text { requeridos del objetivo, con su apoyo, de manera tal que sigan para internalizar } \\
\text { los puntos claves? }\end{array}$ \\
\hline & $\begin{array}{l}\text { - ¿Cómo asegurará que sus estudiantes tendrán múltiples oportunidades de } \\
\text { practicar, con ejercicios que van de fácil a difícil? }\end{array}$ \\
\hline & • ¿Cómo/cuándo realizará una representación para revisar la comprensión? \\
\hline & • ¿Cómo enfrentará los malentendidos? \\
\hline \multirow{3}{*}{$\begin{array}{l}\text { Desarrollo - Práctica } \\
\text { independiente }\end{array}$} & $\begin{array}{l}\text { - ¿Cómo intentarán sus estudiantes los dominios independientes de todo el } \\
\text { conocimiento o habilidades requeridos del objetivo, de manera tal que solidifiquen } \\
\text { su internalización de los puntos claves? }\end{array}$ \\
\hline & • ¿Cómo dará oportunidades para clases de refuerzo y extensión? \\
\hline & $\begin{array}{l}\text { - ¿Cómo resumirán y establecerán sus estudiantes la importancia de lo que } \\
\text { aprendieron? }\end{array}$ \\
\hline Cierre & $\begin{array}{l}\text { - Si la práctica independiente no sirvió como una evaluación, ¿cómo intentarán sus } \\
\text { estudiantes el dominio del conocimiento o habilidades presentadas y practicadas } \\
\text { anteriormente? }\end{array}$ \\
\hline
\end{tabular}


doi: http://dx.doi.org/10.15359/ree.21-2.10

URL: http://www.una.ac.cr/educare

CORREO: educare@una.cr

A continuación, se presentan los recursos de apoyo considerados para implementar la metodología de aprendizaje basado en proyectos y aprendizaje colaborativo, además del desarrollo de las competencias de autoaprendizaje y de trabajo en equipo:

- Elaboración de un set de treinta proyectos de ingeniería civil y ciencias de la ingeniería.

- Elaboración de videos tutoriales para el uso de software MS Excel y MATLAB.

- Preparación de un sistema para la elaboración de bases de datos, foros de consulta y entrega de tareas en un aula virtual. Para el desarrollo de esto se usó la plataforma de la Universidad que se basa en Moodle versión 2.6.

- Elaboración de rúbricas de evaluación de códigos y sistemas de evaluación.

- Elaboración de un instrumento en donde el estudiantado se autoevalúe acerca de los logros de los resultados de aprendizaje de la asignatura.

\section{Ejecución}

En esta etapa se ejecuta lo previamente planificado, considerando el enfoque de este proyecto que es promover y desarrollar el aprendizaje activo, desde dos metodologías simultáneas: el aprendizaje colaborativo y el aprendizaje basado en problemas. Durante las sesiones netamente prácticas se plantean, al estudiantado, las actividades de creación de diagramas de flujos, pseudocódigos y códigos, en donde deben presentar la resolución de un problema de ingeniería, utilizando las herramientas y técnicas revisadas en las clases prácticas/teóricas, los recursos de apoyo (videos tutoriales de MS Excel y MATLAB), y el material bibliográfico del curso. Todos estos productos de salida (informes, diagramas de flujo y códigos) se almacenan en una base de datos disponible en el aula virtual del curso; de esta forma, docente y ayudantes pueden tener acceso a todas las resoluciones de problemas y, a la vez, hacer comentarios y consultas que permitan la mejora y optimización de los productos y del aprendizaje en sí. Para la creación de los productos de salida, el estudiantado cuenta con las rúbricas respectivas, además de contar con la guía y apoyo de su docente y ayudantes de la asignatura.

Cada una de las sesiones de cátedra y taller deben tener una estructura como la presentada en la Tabla 2, para fortalecer y mejorar el aprendizaje. Cada estudiante durante el curso debe presentar la resolución de seis proyectos de ingeniería entregados por el cuerpo académico $y$, además, debe desarrollar un proyecto final propio a partir de un problema o necesidad ingenieril, para posteriormente resolverlo en algún lenguaje de programación. La realización de los seis proyectos y el final se enmarcan en las clases del corte netamente prácticas, tal y como se representa en la Figura 2. 


\section{Evaluación y seguimiento}

Durante todo curso universitario se debe evaluar el logro estudiantil con respecto a cada resultado de aprendizaje que se encuentra plasmado en el programa de la asignatura. Para la consecución de estos se deben evaluar tanto contenidos declarativos como procedimentales.

Los contenidos declarativos son conceptos, datos y hechos; mientras que los procedimentales se refieren a ejecución de procedimientos, técnicas, habilidades, destrezas y métodos. Para evaluar los contenidos declarativos se pueden ocupar, como herramientas: mapas conceptuales, pruebas objetivas, ensayos, y videos. Para evaluar los contenidos declarativos se pueden usar: pruebas de casos, simuladores, juego de roles, y proyectos (Herrera, 2015).

En este curso en particular, los resultados de aprendizaje están en mayor porcentaje asociados a contenidos procedimentales; esto no quiere decir que los contenidos declarativos no deben ser medidos, ya que estos últimos sirven como medida de autocontrol en una etapa inicial de los propios aprendizajes del grupo de estudiantes. Incluso, la generación de diferentes instancias de evaluación y la diversidad en los instrumentos permite al personal docente y a sus estudiantes tomar las medidas correspondientes para mejorar el logro de los aprendizajes (Herrera, 2015).

En la asignatura en la que se aplica el caso de estudio se tienen diversos instrumentos de evaluación, como se presentan en la Tabla 3. Además, en esta tabla se muestran las ponderaciones de cada instrumento con respecto a la nota final del curso.

Tabla 3: Instrumentos de evaluación y ponderación

\begin{tabular}{lll}
\hline Procedimiento evaluativo (tipo de contenido) & Instrumento de evaluación & $\%$ NF \\
\hline Controles escritos (declarativos) & Pauta de evaluación & $8.75 \%$ \\
Tareas (declarativos y procedimentales) & Escala de apreciación & $8.75 \%$ \\
Proyectos (procedimentales) & Rúbrica & $52.50 \%$ \\
Proyecto final (procedimentales) & & $30.00 \%$ \\
\hline
\end{tabular}


doi: http://dx.doi.org/10.15359/ree.21-2.10

URL: http://www.una.ac.cr/educare

CORREO: educare@una.cr

Sin duda, la otra cara de la moneda de cada procedimiento evaluativo es el proceso formativo que se encuentra implícito en cada uno de ellos (Darling-Hammond, 2001; Freire, 2002), específicamente las retroalimentaciones que se pueden realizar tanto de forma escrita como oral. En este curso en particular se consideran como instancias de retroalimentación:

- El propio instrumento evaluativo, como retroalimentación escrita (pauta, escala de apreciación, y rúbrica).

- Retroalimentación oral a todo el grupo curso, específicamente cuando se trata de procedimientos evaluativos, tales como, controles y tareas.

- Retroalimentación oral por grupo de estudiantes, cuando se trata de los proyectos. Como los proyectos tienen tres salidas (diagrama de flujo, código e informe), se considera una instancia de retroalimentación formativa (sin calificación) el diagrama de flujo, previo a la codificación.

\section{Análisis y resultados}

\section{Heteroevaluación}

Para efectos de análisis de la heteroevaluación, se utilizarán los porcentajes de logro en tres de los seis resultados de aprendizaje (RA) del curso:

A. Adquirir habilidades de manejo y análisis de datos en MS Excel (Figura 3a).

B. Adquirir el dominio básico del manejo y análisis de datos en MATLAB (Figura 3b).

C. Utilizar herramientas como MS Excel y MATLAB para la resolución de problemas de ingeniería (Figura 3c). 
doi: http://dx.doi.org/10.15359/ree.21-2.10

URL: http://www.una.ac.cr/educare

CORREO: educare@una.cr

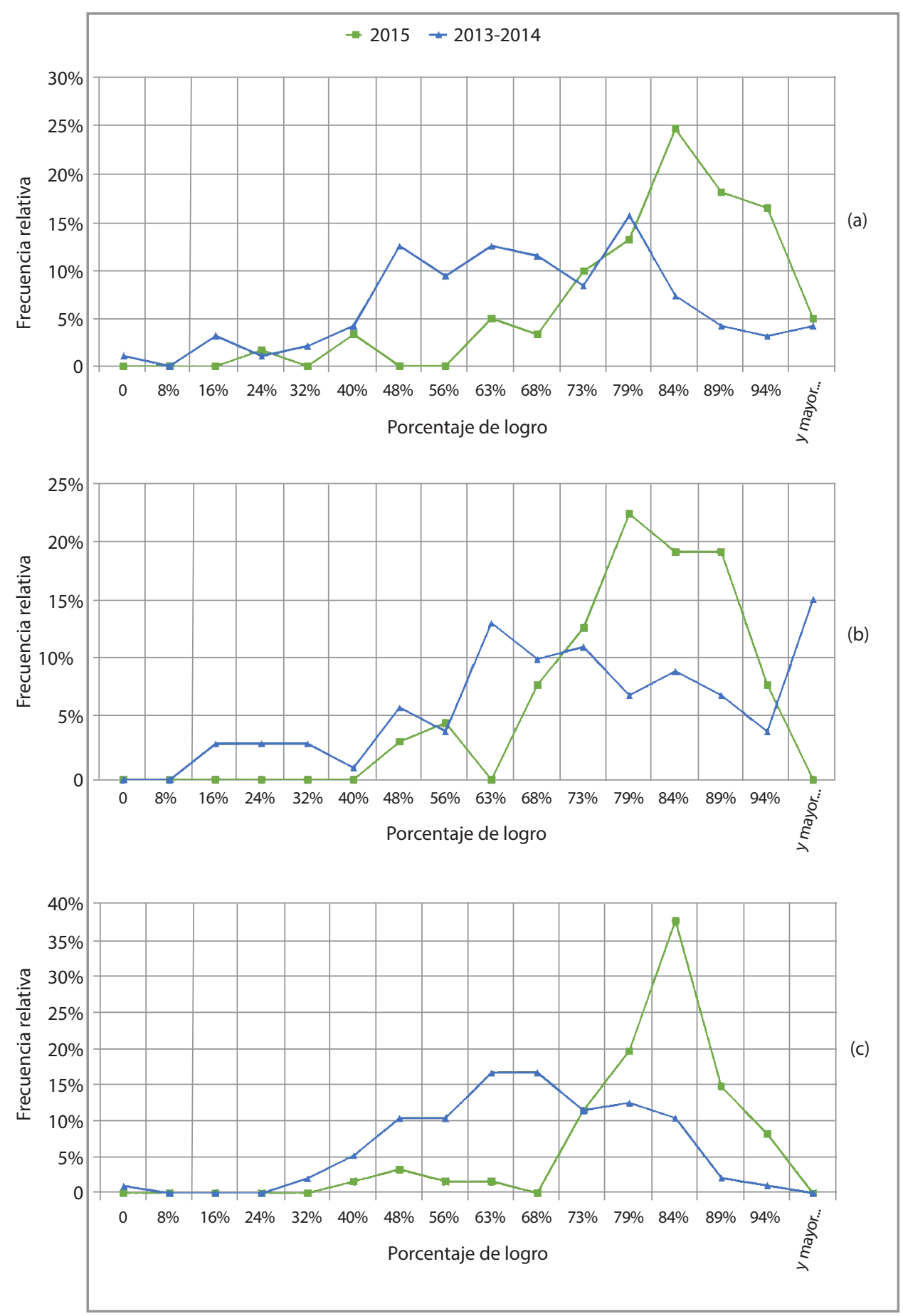

Figura 3: Logros obtenidos a partir de la heteroevaluación. 
doi: http://dx.doi.org/10.15359/ree.21-2.10

URL: http://www.una.ac.cr/educare

CORREO: educare@una.cr

De los resultados obtenidos para los tres RA se puede apreciar una tendencia significativa de un mayor porcentaje de logro, por parte del estudiantado, cuando se aplica la metodología de aprendizaje activo (2015) en desmedro de la metodología de aprendizaje tradicional (20132014) de clase catedrática. Lo anterior se puede apreciar comparando las medidas de tendencia central y las medidas de dispersión de cada uno de los resultados de aprendizaje (Tabla 4), presentado diferencias más significativas en los RA A y C.

Tabla 4: Medidas de tendencia central y dispersión heteroevaluación

\begin{tabular}{l|c|c|c|c|c|c}
\hline \multirow{2}{*}{ Resultado de aprendizaje } & \multicolumn{2}{c|}{ Media } & \multicolumn{2}{c|}{ Mediana } & \multicolumn{2}{c}{ Coeficiente de variación } \\
\cline { 2 - 7 } & $2013-2014$ & 2015 & $2013-2014$ & 2015 & $2013-2014$ & 2015 \\
\hline $\begin{array}{l}\text { A. Adquirir habilidades de manejo y } \\
\text { análisis de datos en MS - Excel }\end{array}$ & $65.22 \%$ & $79.25 \%$ & $69.33 \%$ & $82.22 \%$ & $32.13 \%$ & $17.71 \%$ \\
\hline $\begin{array}{l}\text { B. Adquirir el dominio básico del manejo } \\
\text { y análisis de datos en MATLAB }\end{array}$ & $75.10 \%$ & $76.01 \%$ & $74.67 \%$ & $78.22 \%$ & $27.70 \%$ & $18.84 \%$ \\
\hline $\begin{array}{l}\text { C. Utilizar herramientas como MS - } \\
\text { Excel y MATLAB para la resolución } \\
\text { de problemas de ingeniería }\end{array}$ & $66.23 \%$ & $78.06 \%$ & $65.78 \%$ & $79.33 \%$ & $21.13 \%$ & $14.13 \%$ \\
\hline
\end{tabular}

Con respecto a la forma de las gráficas, se puede apreciar que cuando se aplica una metodología de enseñanza tradicional (2013-2014) los resultados de la evaluación tienen una distribución parecida a una normal estándar, mientras que cuando se aplica una metodología de aprendizaje activo, la distribución normal se carga hacia la derecha, evidencia también del aumento del logro estudiantil en los resultados de aprendizaje.

Para los tres resultados de aprendizaje medidos en la heteroevaluación se aprecia una baja del coeficiente de variación cuando se aplican metodologías activas; esto explica que el aumento del porcentaje de logro no se da solamente por una parte de estudiantes que destacan y arrastran el promedio curso hacia un valor más positivo, sino que está asociado al aumento del logro de todo el grupo curso.

\section{Autoevaluación}

Para efectos de la autoevaluación, se le pidió al estudiantado que se calificara a sí mismo, individualmente, en una escala de 1 a 5 , con respecto a su nivel de logro de cada uno de los seis resultados de aprendizaje del curso. Los resultados de la calificación que se adjudica cada estudiante se representa en la Figura 4, en donde se evidencia la frecuencia relativa de cada grupo en estudio. Por otro lado, en la Figura 5 se muestra la media(a) y el coeficiente de variación (b) de cada grupo en estudio con respecto a cada uno de los resultados de aprendizaje del curso. 
doi: http://dx.doi.org/10.15359/ree.21-2.10

URL: http://www.una.ac.cr/educare

CORREO: educare@una.cr
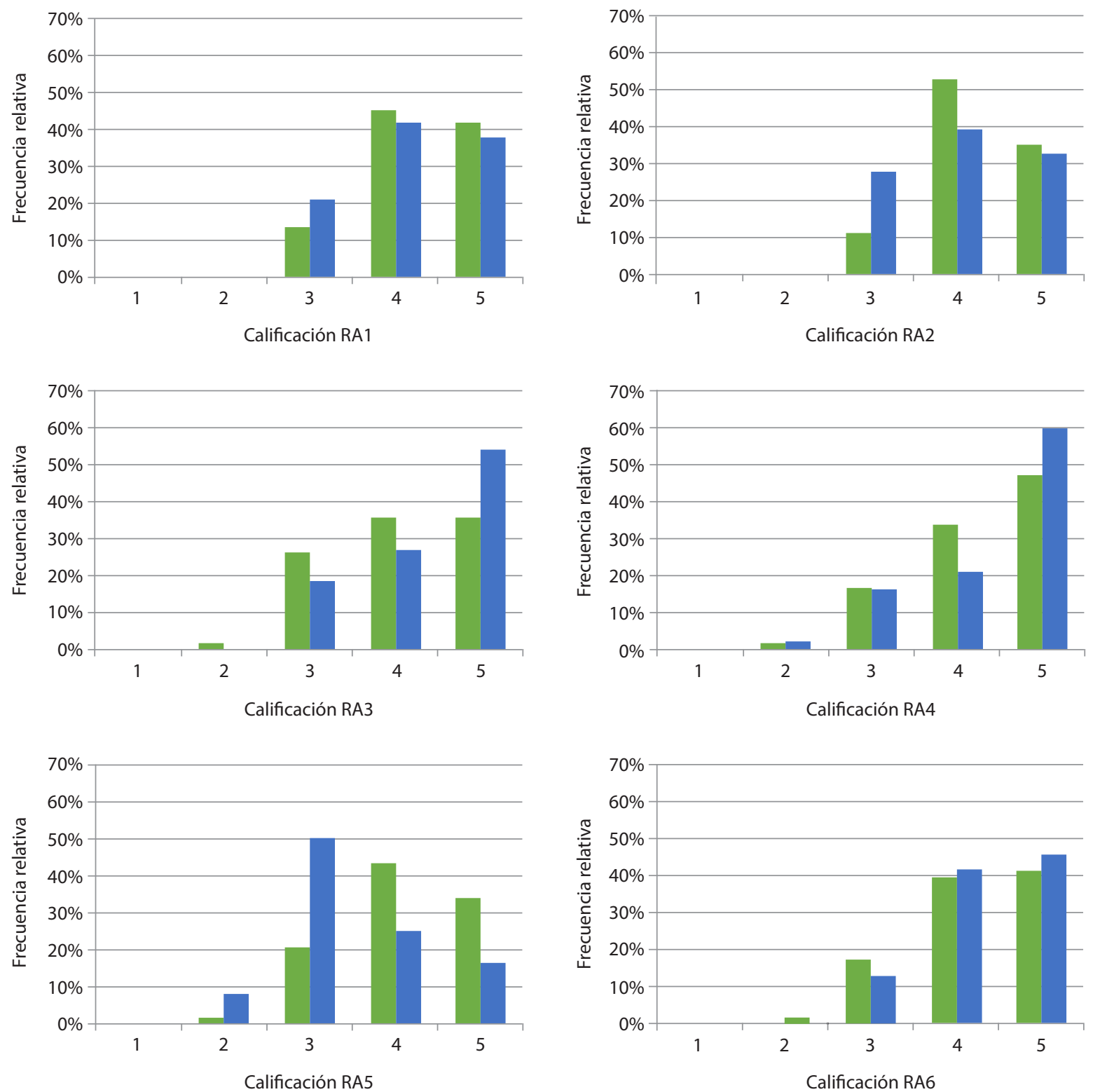

Figura 4: Autoevaluación. 
doi: http://dx.doi.org/10.15359/ree.21-2.10

URL: http://www.una.ac.cr/educare

CORREO: educare@una.cr

(a)

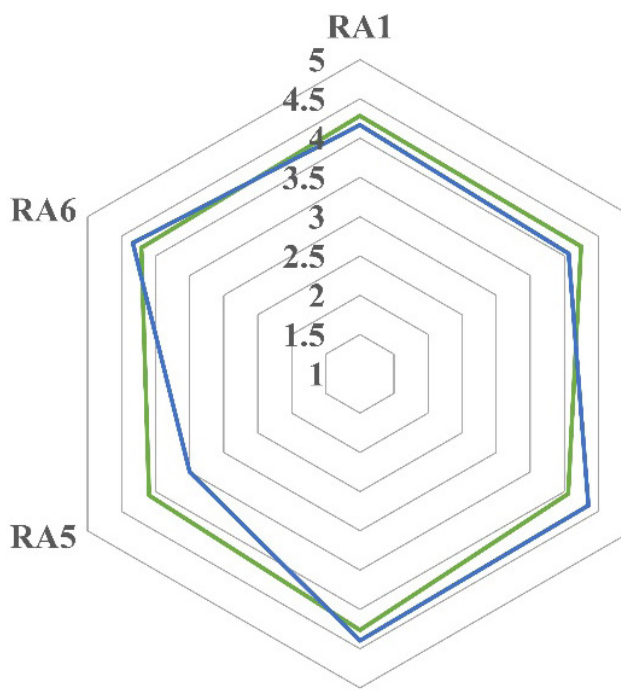

RA4
$-2015-2013-2014$

RA2

RA3

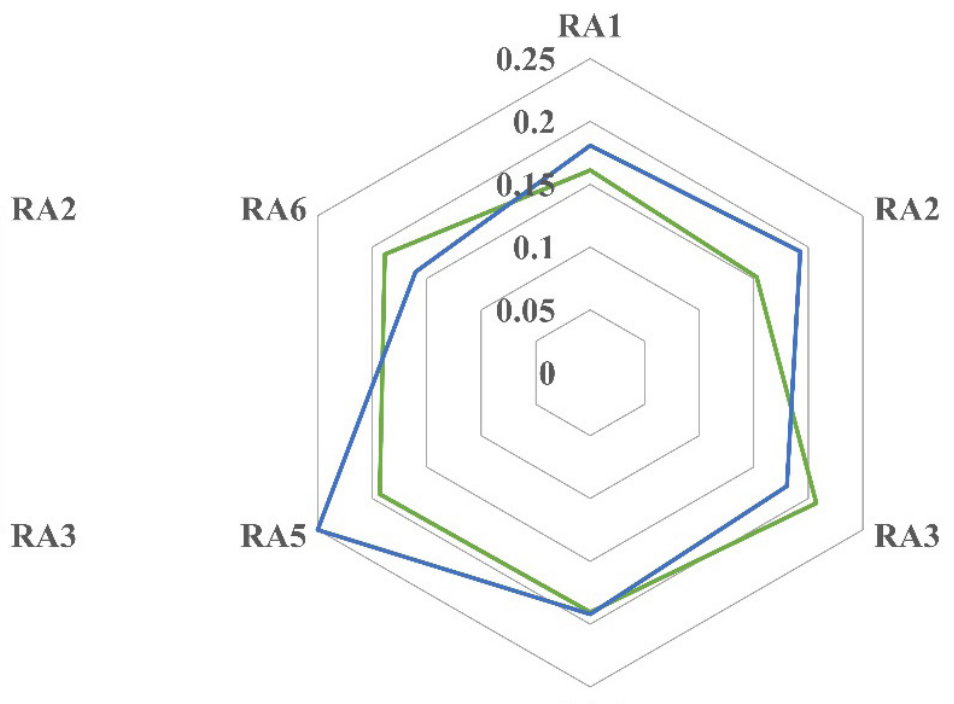

RA4

(b)

Figura 5: Media (a) y coeficiente de variación(b) autoevaluación.

De las Figuras 4 y 5 , se puede desprender que el estudiantado se autoevalúa de una forma bastante similar cuando se le aplica cualquiera de las dos metodologías de enseñanza comparadas en este estudio.

Sin embargo, el resultado de aprendizaje con diferencias más considerable entre un grupo y otro es el de "Generar informes de ingeniería mediante el análisis de datos", esto se debe únicamente al cambio de los instrumentos de evaluación asociado a cada metodología, desde la clásica prueba de 90 minutos, al trabajo de una semana en donde el entregable final es un código respaldado de un informe.

Si se traducen las calificaciones de la autoevaluación a porcentajes se obtiene que en promedio se califican entre $62.50 \%$ y $84.90 \%$ de logro los años 2013-2014 en donde se aplicó una metodología tradicional y entre $77.36 \%$ y $82.08 \%$ de logro cuando se aplicó una metodología de aprendizaje activo. Si bien, en ambos casos el porcentaje de logro de la autoevaluación supera al promedio de la heteroevaluación, la brecha existente entre la hetero y autoevaluación es significativamente menor cuando se aplican técnicas e instrumentos de evaluación de aprendizaje activo, lo que hace concluir que estas herramientas son más justas al momento de evaluar los distintos aprendizajes del curso (Tabla 5). 
Tabla 5: Diferencia entre hetero y autoevaluación

\begin{tabular}{|c|c|c|c|c|c|c|}
\hline \multirow{3}{*}{ Resultado de aprendizaje } & \multicolumn{3}{|c|}{ 2013-2014 (Tradicional) } & \multicolumn{3}{|c|}{2015 (Activo) } \\
\hline & Media & Media & \multirow{2}{*}{ Diferencia } & Media & Media & \multirow{2}{*}{ Diferencia } \\
\hline & Hetero & Auto & & Hetero & Auto & \\
\hline $\begin{array}{l}\text { A. Adquirir habilidades de manejo y análisis de } \\
\text { datos en MS - Excel }\end{array}$ & $65.22 \%$ & $83.85 \%$ & $18.63 \%$ & $79.25 \%$ & $76.42 \%$ & $2.83 \%$ \\
\hline $\begin{array}{l}\text { B. Adquirir el dominio básico del manejo y } \\
\text { análisis de datos en MATLAB (Figura 4b). }\end{array}$ & $75.10 \%$ & $84.90 \%$ & $9.80 \%$ & $76.01 \%$ & $81.60 \%$ & $5.59 \%$ \\
\hline $\begin{array}{l}\text { C. Utilizar herramientas como MS - Excel y } \\
\text { MATLAB para la resolución de problemas de } \\
\text { ingeniería (Fiqura 4c) }\end{array}$ & $66.23 \%$ & $83.33 \%$ & $17.10 \%$ & $78.06 \%$ & $80.19 \%$ & $2.13 \%$ \\
\hline
\end{tabular}

\section{Ventajas y desventajas de la metodología de aprendizaje activo}

De los comentarios obtenidos de estudiantes del curso 2015, acerca de la metodología de aprendizaje activo (aprendizaje basado en proyectos + aprendizaje colaborativo), se destacan los siguientes comentarios:

- Aprender con el propio ritmo de cada estudiante y no bajo una estructura rígida de contenidos clase a clase.

- Aprender de forma natural con base en el ensayo y error. El error no es un castigo como en la metodología tradicional, sino una forma de profundizar el aprendizaje.

- Trabajar en equipo le da mayor efectividad al aprendizaje, de la discusión entre dos o más personas se genera mayor conocimiento, "cuando uno le tenía que explicar a un compañero no solo aprendía él sino que también yo aprendía más".

- Poner en práctica los conocimientos en problemas reales de ingeniería.

- Aprender por sus propios medios, no se les entrega todo con facilidad, muchos temas tienen que investigar ellas o ellos mismos y con el apoyo de sus pares. Evidencia de esto se encuentra en el trabajo de Astudillo, Navarrete, Jara y Faúndez (2015), en donde explican cómo el autoaprendizaje se manifiesta espontáneamente en el aula cuando existe un comportamiento colectivo.

- Solicitar apoyo del profesorado y sus ayudantes para resolver dudas, "ambos no te entregaban las respuestas, sino que te guiaban para encontrarlas uno mismo". 
doi: http://dx.doi.org/10.15359/ree.21-2.10

URL: http://www.una.ac.cr/educare

CORREO: educare@una.cr

Si bien son variadas las ventajas que cuenta el estudiantado, también existen algunas desventajas como se presentan a continuación:

- El tiempo en una evaluación no se acotaba; sin embargo, el que se tenía que invertir para desarrollar los proyectos era bastante alto en comparación con un curso normal.

- Algunos proyectos de ingeniería implicaban un gasto de tiempo importante en investigación y solo entender el problema, antes de comenzar a hacer el algoritmo.

- Ponerse de acuerdo con el resto de los miembros del equipo de trabajo era complicado, sobretodo en temporada de evaluaciones.

\section{Conclusiones}

De acuerdo con los resultados obtenidos, es posible extraer las siguientes conclusiones y recomendaciones:

- Cuando se aplican metodologías de aprendizaje activo se aumenta el logro de los resultados de aprendizaje por parte de los grupos de estudiantes, en comparación cuando se aplican metodologías de enseñanza y de evaluación tradicional, tales como clases catedráticas y pruebas en 90 minutos. Esto, medido a partir de la heteroevaluación, es decir, a partir del uso de rúbricas, pruebas y pautas, según corresponda la instancia evaluativa.

- La percepción de logro de los propios grupos de estudiantes no varía significativamente según la metodología de enseñanza de aprendizaje que se aplique al grupo curso. Por otro lado, la brecha que existe entre la autoevaluación y la heteroevaluación se reduce cuando se aplican metodologías de aprendizaje activo, como es en este caso de estudio.

- La aplicación de metodologías de aprendizaje activo, tales como el aprendizaje basado en proyectos y el aprendizaje colaborativo, implican una mayor inversión de tiempo de estudiantes y docente a cargo. En el primer caso, el estudiantado debe reunirse y trabajar en equipo, realizar proyectos, avanzar a su propio ritmo, lo que implica una mayor inversión de tiempo a lo largo de todo el curso, en comparación con la metodología tradicional de enseñanza y evaluación del tipo pruebas, en donde los esfuerzos de tiempo se concentran en las cercanías de las instancias de evaluación solamente. Por otro lado, los cuerpos docentes deben invertir un mayor tiempo debido a la preparación del material apto para el modelo de enseñanza (proyectos, ejercicios, tareas, rúbricas), estar siempre disponible para guiar el aprendizaje y no entregar solo un resultado, realizar un trabajo más personalizado debido a que los ritmos de aprendizaje son distintos para cada persona, y generar instancias de retroalimentación continua con sus estudiantes. 
- El aprendizaje activo fomenta de mayor forma habilidades blandas, como la generación de informes y reportes de trabajo; a diferencia de las metodologías tradicionales. Esto se debe a que los instrumentos de evaluación cambian considerablemente entre la entrega de un informe o reporte y una prueba. Sin duda, esta habilidad inicia a los grupos estudiantiles en una de labor que deberán ejecutar en su desempeño profesional.

- El aprendizaje basado en proyectos hace que el estudiantado esté más motivado por su aprendizaje, sobre todo cuando estos proyectos son problemas reales de su área (en este caso ingeniería) que tendrá que resolver durante su formación en pregrado y su desarrollo profesional.

- El aprendizaje colaborativo genera varias ventajas, entre las cuales se pueden nombrar: desarrollo de habilidades de alto nivel cuando se enseñan mutuamente compañeros y compañeras, aumento de la percepción de que las demás personas de la clase se preocupan por su aprendizaje y quieren ayudar.

\section{Agradecimientos}

Mis agradecimientos a mis estudiantes, a mis ayudantes y a la Unidad de Mejoramiento de la Docencia Universitaria (UMDU) de la Pontificia Universidad Católica de Valparaíso por apoyar y financiar este trabajo con el proyecto 2015.FIN2030.EIC.01.

\section{Referencias}

Astudillo, H. F., Navarrete, M. A., Jara, C. I. y Faúndez, C. A. (2015). Evidencia de auto aprendizaje como manifestación de un comportamiento colectivo espontáneo en el aula. Formación Universitaria, 8(1), 43-50. doi: https://doi.org/10.4067/S0718-50062015000100006

Biggs, J., \& Tang, C. (2011). Teaching for quality learning at university (4a ed.). Buckingham: McGraw-Hill.

Darling-Hammond, L. (2001). El derecho de aprender. Crear buenas escuelas para todos. Barcelona: Ariel.

Pontificia Universidad Católica de Valparaíso (PUCV). (2015). Informe de autoevaluación para acreditación. Escuela de Ingeniería Civil, Pontificia Universidad Católica de Valparaíso, Chile.

Pontificia Universidad Católica de Valparaíso, Escuela de Ingeniería Civil. (2014). Programa de asignatura Informática Aplicada (CIV-110). Chile, Valparaíso. Recuperado de http://media. wix.com/ugd/bc004c 3256b165210244cb83d908e5c38fd4d2.pdf 
doi: http://dx.doi.org/10.15359/ree.21-2.10

URL: http://www.una.ac.cr/educare

CORREO: educare@una.cr

Freire, D. E. (2014). La conducta disruptiva y su incidencia en el rendimiento académico de las/ os estudiantes de los $10^{\mathrm{mo}}$ grados de educación general básica paralelos "I, II" del colegio universitario "Juan Montalvo", de la provincia de Tungurahua, cantón Ambato (Informe final del trabajo de graduación). Universidad Técnica de Ambato, Ecuador. Recuperado de http://repo.uta.edu.ec/bitstream/123456789/12416/1/FCHE-PSEP-104.pdf

Freire, P. (2002). Pedagogía de la autonomía. Saberes necesarios para la práctica educativa (2ª ed.). Buenos Aires: Editorial Siglo Veintiuno.

Herrera, R. F. (Octubre, 2015). Diversidad en los instrumentos de evaluación: Caso práctico y valorización estudiantil. En XXVIII Congreso chileno de educación en ingeniería (pp. 1-8), Copiapó, Chile. Recuperado de https://www.academia.edu/20063292/DIVERSIDAD EN LOS INSTRUMENTOS DE EVALUACI\%C3\%93N CASO PR\%C3\%81CTICO Y VALORIZACI\%C3\%93N ESTUDIANTIL

Huber, G. L. (2008). Aprendizaje activo y metodologías educativas. Revista de Educación, número extraordinario, 59-81. Recuperado de http://www.revistaeducacion.mec.es/re2008/ re2008 04.pdf

Johnson, D. W. \& Johnson, R. T. (2000). Teaching students to be peacemakers: Results of twelve years of research. Paper presented at the Society for the Psychological Study of Social Issues Convenition, Minneapolis, MN.

Maldonado, M. (2008). Aprendizaje basado en proyectos colaborativos. Una experiencia en educación superior. Laurus Revista de Educación, 14(28), 158-180. Recuperado de http:// www.redalyc.org/pdf/761/76111716009.pdf

Maldonado, M. y Vásquez, M. (2008). Experiencia de desarrollo de un proyecto de aprendizaje colaborativo como estrategia formativa. Educación Médica Superior, 22(1). Recuperado de http://www.bvs.sld.cu/revistas/ems/vol22 1 08/ems01108.htm

Martínez, J. A. (2011). La empleabilidad: Una competencia personal y una responsabilidad social. Contribuciones a las Ciencias Sociales. Recuperado de http://www.eumed.net/rev/ cccss/15/jamg.html

Serrano, J. M. y Calvo, M. T. (1994). Aprendizaje cooperativo: Técnicas y análisis dimensional. Murcia: Caja Murcia, Obra Cultural.

Tyler, R. W. (1949). Basic principles of curriculum and instruction. Chicago: University of Chicago Press. 